\title{
AI and Machine Learning in Retail Pharmacy: Systematic Review of Related Literature
}

\section{Praveen Kumar Donepudi}

Principal Architect, IT Infrastructure Services, Cognizant Technology Solutions, United States

\begin{abstract}
Artificial intelligence and machine learning are the future of every field. These can be applied in any field for the better or efficient performance. Both these can be used in retail pharmacy as a solution to different problems. The machine learning prediction model can help in predicting the disease of patients and it can also be used to predict the medicine for the patient. AI systems can be used to automate the tasks that will help in saving the time and also the tasks will be performed by using the less resources.
\end{abstract}

Keywords: Artificial intelligence, retail pharmacy, prediction algorithms, machine learning

\section{INTRODUCTION}

Retail pharmacy is a kind of pharmacy that can be considered as a shop. Through this shop different persons can buy the medicines according to their requirements. Retail pharmacy is also known as the community pharmacy where a community pharmacist works to serve the people in the form of providing required medicines. Community pharmacist also guides the people about the use of medicines. Artificial Intelligence and machine learning are the future of every field. As like the banks, industries, the artificial intelligence should also be used in pharmacy. Most people think that the machine learning and artificial intelligence are the same. But these are not the same in actual there is a little difference. Machine learning algorithms are used by the artificial intelligence devices to perform the actions.

The traditional system of health care and pharmacy is not suitable to be adopted in the modern time. In (Iyawa et al., 2017) the researchers said that the current health care system must be changed to respond to the emerging crisis. Sometimes there is a shortage of drugs on pharmacy stores which is a big risk to the health of the people. There is a need for a prediction about the upcoming shortage of the medicines. This shortage is also created when the medicines are stolen by different persons that can be the nurses, pharmacists etc. There should be a way to keep the medicines secure from these type of persons. Most of the time the prescriber recommends the medicine that is known to him instead of the medicine that is most effective. There is a need for a system that can tell about the medicines that are most effective against a disease. 
In the past, different researchers have worked for the importance of Artificial intelligence and machine learning. The researcher has shown how the AI and machine learning can be helpful in reducing the work of a human being. In (Donepudi, 2017a) the researchers said that the main aim of machine learning and Artificial intelligence is to minimize the work load of a person. They said that the AI and machine learning should be used in different fields especially in retailing because this has low profit margin and more human work. The researchers in (Cassel, 2012) said that the retail clinics are very helpful for the community because a number of patients are handled by them. There are a number of retail clinics available that helps the patients to get the required medicine easily. The burden on the hospitals is getting down because of these retail clinics. But the main challenging thing is the attachment of these retail clinics with the health care system. This will help them to get all the data of a patient while giving him the medicine.

This literature review is based on different research papers to show the importance of artificial intelligence and machine learning for the field of retail pharmacy. The work of different researchers will be discussed in the favor of AI and machine learning.

Further sections of this literature review include the methodology in section 2, research question in section 3, Search Process in section 4, result and discussion in section 5, and the conclusion in section 6 .

\section{Methodology}

The methodology that is adopted for this literature review is the systematic literature review. About twenty papers were found against the search string.

\section{Inclusion Exclusion}

Some of the papers were not relevant to be added in this literature review. That's why these are ignored. There was an inclusion and exclusion criteria for the papers to be included. This criteria ensures that the journal paper must be written in good English and relevant to the topic of this literature review. Eleven of the twenty found papers fulfilled the criteria to be included in this literature review.

\section{Quality Assessment}

The quality of the papers is assessed on the base of the research work they present. The research papers that have the similar or relevant content to the topic are included.

\section{Research Question}

The main part of the literature review is the research question. These can help the reader to understand the founding of a literature review. The research questions that will be answered in this literature review are given below.

1. How Artificial Intelligence saves the time and resources in the retail pharmacy?

2. How machine learning algorithms prediction can be helpful?

\section{Search Process}

The data for this literature review is found from different databases. These include the famous journal databases such as Elsevier, ACM, IEEE Xplore etc. Different methods are used at different phases to find out the most relevant research papers. First of all the research papers are selected on the basis on their titles and abstracts. Then in the second 
phase the complete content and the conclusion of the research paper is checked to include it in this literature review.

\section{Result ANd Discussion}

Artificial intelligence is the technology that is making changes in every field. These changes are actually the improvements by the artificial intelligence. Artificial intelligence device can learn from the environment (Michalski et al., 1983) and this is one of the most important attribute of artificial intelligence. These devices can make the action according to their learning. Machine learning algorithms are used to train these artificial intelligence devices. Every field will be using artificial intelligence in the future. Because the artificial intelligence is very helpful to increase the performance.

RQ 1: How Artificial Intelligence saves the time and resources in the retail pharmacy?

Use of artificial intelligence is increasing day by day. All the fields are trying to use artificial intelligence for the better results or performance. AI is beneficial for every field or organization whether it is the banking sector or health. The main advantage of using the artificial intelligence is the saving of time and resources. Different tasks can be performed without human involvement. The artificial intelligence machines can perform these tasks in an efficient way. In (Donepudi, 2016) the researchers proposed a software for the manufacturers that will help in delivering the medicines in cost effective and timely manner. This software uses the artificial intelligence to design, automate, analyze, and monitor human centric and integration centric activities. The authors in (Vyas et al., 2018) in said that the artificial intelligence has many advantages in the field of pharmacy. One of the major advantages is that it reduces the time utilized for making a medicine. This will help in making more medicines in less time. Through the use of Artificial intelligence a good quality medicine can be made in less cost. Money is one of the main resources of a company which must be utilized in a proper way. The use of Artificial intelligence is also helpful for the patients. In (Cai et al., 2016) the author said that through the artificial intelligence the patients can be treated in a good way. Moreover, artificial intelligence can also be used for better treatment in less time.

RQ 2: How machine learning algorithms prediction can be helpful?

There are different machine learning algorithms for the prediction purpose. This includes the Support vector machine, K-Nearest Neighbors, Linear regression, Logistic regression etc. These algorithms are first trained through a train data set. This training data set helps the algorithm to predict the result for an unseen data set. This prediction is based on the training data set. In the field of retail pharmacy the machine learning algorithms can be proved helpful because of their prediction. This training data set also requires the outcomes so that it can predict what happens to the patient (Vyas et al., 2018). One of the issues in the fields of health is finding the disease of the patient. This issue can be resolved through the machine learning prediction algorithms. In (Chen, Hao, Hwang, Wang, \& Wang, 2017) authors said that the machine learning algorithms can be used to predict the patient's disease accurately. Sometimes it also happens that the medicines are not available in pharmacy for the patients. In (Donepudi, 2017b) the authors said that it is possible to predict the demand through the machine learning models. This can be done by training the model with previous year data set and then the model will predict the demand of the upcoming year. If there is a chance of shortage then the more medicines can be prepared. Machine learning algorithms can also be used for the monitoring and 
detection of drugs diversion. The medicines are sometime theft by different persons that can be the nurses, physicians, pharmacist etc.

The researchers in (Chen et al., 2017) said that the machine learning prediction models can be used to predict the patients that are at most risk. This can help in giving them more attention and more care. Machine learning prediction algorithms can also predict the medicine for a patient according to the training data set given to it.

The AI and machine learning also minimize the chance of mistakes in storing the data. It can also help in providing the patient's data to the pharmacist so that the correct medicine can be given to the patients.

\section{CONCLUSION}

The study shows the importance of artificial intelligence and the machine learning algorithms. It is clearly described how the artificial intelligence can be used for saving the time as well as the resources. The retail pharmacy must have to adopt this for the better performance. Instead of giving the known medicine, the pharmacist can provide the effective medicine to the patient with the help of machine learning prediction algorithms. These algorithms can be used for predicting the disease of a patient and can also be used for predicting the medicine for that disease. The future work can be the implementation of these machine learning algorithms so that the correct medicine can be provided to the patients. This will save the time and will provide the more accuracy.

\section{RefEREnCES}

Cai, Y., Dai, D., \& Hua, S. (2016). Using machine learning algorithms to improve the prediction accuracy in disease identification: An empirical example. Athens: The Steering Committee of The World Congress in Computer Science, Computer Engineering and Applied Computing (WorldComp). Retrieved from https:// search.proquest.com/docview/1806429009?accountid=35493

Cassel, C. K. J. J. (2012). Retail clinics and drugstore medicine. 307(20), 2151-2152.

Chen, M., Hao, Y., Hwang, K., Wang, L., \& Wang, L. J. I. A. (2017). Disease prediction by machine learning over big data from healthcare communities. 5, 8869-8879.

Donepudi, P. (2016). Influence of Cloud Computing in Business: Are They Robust? Asian Journal of Applied Science and Engineering, 5(3), 193-196. https://doi.org/10.5281/zenodo.4110309

Donepudi, P. (2017a). AI and Machine Learning in Banking: A Systematic Literature Review. Asian Journal of Applied Science and Engineering, 6(3), 157-162. https://doi.org/10.5281/zenodo.4109672

Donepudi, P. K. (2017b). Machine Learning and Artificial Intelligence in Banking. Engineering International, 5(2), 83-86. https://doi.org/10.18034/ei.v5i2.490

Iyawa, G. E., Herselman, M., \& Botha, A. (2017). A scoping review of digital health innovation ecosystems in developed and developing countries. Piscataway: The Institute of Electrical and Electronics Engineers, Inc. (IEEE). Retrieved from https://search.proquest.com/docview/1962316664?accountid=35493

Michalski, R.S., Carbonell, J.G., Mitchell, T.M. (1983). Machine Learning: An Artificial Intelligence Approach. Springer, https://www.springer.com/gp/book/9783662124079

Vyas, M., Thakur, S., Riyaz, B., Bansal, K. K., Tomar, B., \& Mishra, V. J. A. J. P. (2018). Artificial intelligence: the beginning of a new era in pharmacy profession. 12(2), 72-76. 